\title{
Embarazo ectópico avanzado
}

\author{
REVISION DE LA LITERATURA COLOMBIANA \\ $Y$ PRESENTACION DE 8 NUEVOS CASOS * \\ Dr. Jarme Botero-Uribe
}

Del Departamento de Obstetricia y Ginecología de la Facultad de Medicina de la Universidad de Antioquia.

\section{INTRODUCCION}

El presente trabajo ha sido elaborado utilizando el material bibliográfico de la biblioteca de la Facultad de Medicina, constituído por revistas colombianas, muchas de las cuales son colecciones incompletas, tienen un deficiente índice de sus volúmenes o carecen totalmente de él. Además, por el material clínico recopilado en el servicio de Obstetricia del Hospital Universitario de San Vicente de Paúl de Medellín y en el Instituto de Anatomía Patológica, en los últimos 11 años. Uno de los casos que presentamos nos ha sido enviado como una comunicación personal (17). Se seleccionaron para esta revisión los fetos correspondientes a embarazos de 28 o más semanas, de $1.500 \mathrm{gm}$. o más de peso, o que por la historia se supuso que corresponderían a embarazos a término.

\section{CASOS DE LA LITERATURA COLOMBIANA}

En 1912 el Dr. Pompilio Martínez presentó ante la Academia Nacional de Medicina (1) el primer caso, de que se tiene noticia en esta revisión, de embarazo extrauterino a término, el cual permaneció dentro del abdomen por cuatro meses más después de que la paciente había llegado a la fecha probable del parto. Con

* Presentado en el IV Congreso Colombiano de Obstetricia y Ginecología. - Barranquilla, $195 \mathrm{C}$. 
el diagnóstico correcto se practicó una laparotomía y se encontró un feto a término muerto, dentro de la trompa izquierda enormemente distendida; la placenta se implantaba en la pared posterior de este saco. La intervención consistió en una histerectomía y una salpingectomía bilateral y ooforectomía derecha y la pieza operatoria fue presentada a la Academia de Medicina. La paciente recuperó de manera satisfactoria.

El segundo caso fué publicado en 1914 por el Dr. Anselmo Gaitán (2) y ocurrió en una paciente con antecedentes de pelviperitonitis, en la cual el embarazo progresó satisfactoriamente hasta alcanzar el término, pero el parto no ocurrió dentro del tiempo que estaba previsto y la paciente fue hospitalizada. Se hizo el diagnóstico de embarazo intrauterino y de adherencia de las membranas al útero. La laparotomía practicada dos meses después del término permitió obtener un feto muerto, situado entre las hojas del ligamento ancho a donde se supuso había llegado después de su implantación inicial en la porción ampular de la trompa. Al parecer se hizc extirpación del embarazo y de la trompa derecha; la paciente permaneció en shock después del acto operatorio y murió al día siguiente.

En 1917 publicó el Dr. Gil J. Gil (3) dos casos de embarazo ectópico, uno de los cuales había llegado al término y fue intervenido después de permanecer 11 meses más en el abdomen, según se colige por los datos de la historia. El diagnóstico preoperatorio fue el de embarazo extrauterino con hematocele pelviano o quiste del ovario y fibroma subperitoneal. A la laparotomía se encontró un embarazo intrauterino de 4 meses y un embarazo intraligamentario a término con feto muerto pero bien conservado. Se hizo una extirpación del embarazo y una histerectomía, que confirmó el embarazo intrauterino. La paciente tuvo un postoperatorio normal.

El Dr. José M. Uribe G. (4), siendo aún estudiante, publicó en 1921 un caso observado en el servicio del Profesor Montoya y Flórez de una paciente con 18 meses de amenorrea y a quien en la laparotomía se encontró un embarazo abdominal, sin que se den detalles sobre su localización ni estado de conservación. La paciente se recuperó satisfactoriamente del acto operatorio.

El Dr. Roberto Serpa (5), en Bucaramanga, hizo por primera vez el diagnóstico de embarazo ectópico con feto vivo a térmi- 
no y obtuvo éste en perfectus condiciones después de una laparotomía mediana bajo anestesia con cloroformo. Por haberse presentado desprendimiento accidental de la placenta hizo la extracción de ésta y logró hemostasia satisfactoria. La paciente murió en el tercer día postoperatorio sin haberse recuperado totalmente del shock producido por el actc quirúrgico. El niño pesó $3.500 \mathrm{gm}$. y dos años después demostraba un desarrollo normal.

El sexto caso fue publicado por el Dr. Rafael Ucrós (6) en 1927 y corresponde a una paciente a quien se había practicado antes una cesárea corporal. Tuvo amenorrea de nueve meses, sintió movimientos fetales y luego permaneció un tiempo no determinado sin siinguna sintomatología. La laparotomía demostró un litopedio de :mos ocho meses adherido a la cicatriz de la antigua cesárea que presentaba una zona de dehiscencia, y un embarazo de ocho seminas en el interior de la cavidad uterina. Se practicó una histerectomía subtotal con extirpación del litopedio y la paciente recuperó en forma satisfactoria. Uno de nuestros casos, el $\mathrm{N}^{0} 7$, es similar a éste.

El Dr. Roberto Serpa (7) publicó el séptimo caso, correspondiente a un embarazo tubario a término con feto muerto; hizo extirpación del saco y la paciente recuperó normalmente.

En 1940 (8) el mismo autor publicó un recuento sobre embarazo abdominal e incluyó el primer caso operado por él con obtención de feto vivo, al cual agregó: un litopedio con feto a término, un embarazo que llegó a término sin que se produjera el parto y en el cual cinco meses más tarde ocurrió la expulsión de huesos a través de una fístula que se formó en el ombligo, y por último, un litopedio óue fue extraído seis años después de un embarazo que llegó a término y no se siguió del parto.

El Dr. Juan Barrios Z. publicó en 1940 cuatro observaciones más (9) ; una de ellas correspondía a una paciente con eclampsia a la cual se hizo una cesárea indicada por la gravedad de la toxemia y se encontró accidentalmente un feto que se extrajo y alcanzó a vivir dos meses. La madre murió en el acto operatorio a consecuencias de la toxemia y la hemorragia que acompañó a la intervención. Es éste el segundo caso hallado en el cual el niño extraído de la cavidad abdominal, sobrevivió por algún tiempo. Los casos restantes corresponden todos a fetos que murieron antes de practicar la laparotomía. 
Los casas publicados por el Dr. Rafael Ramírez M. (10), tienen la peculiaridad de que se presentaron con muy corto intervalo, pues solo dos días transcurriercn entre la presentación del uno y del otro. El examen microscópico de la segunda pieza demostró muy probablemente un embarazo ovárico, localización que es extremadamente rara. Ambos fetos estaban muertos; las pacientes recuperaron en forma satisfactoria.

El Dr. Guillermo Navas A. (18) publicó un caso de embarazo tubario izquierdo a término atendido por el Dr. Jorge Solanilla en Cali, (11) en el cual se hizo diagnóstico correcto preoperatorio y se obtuvo un feto vivo. La madre se recuperó bien. El mismo autor relata un caso publicado por el Dr. Ramírez M., (12) en el cual se hizo el diagnóstico preoperatorio correcto de embarazo tuboovárico.

Los casos restantes son muy similares a los anteriores, y están incluídos, en orden de publicación, en el cuadro 1.

\section{CUADRO 1}

Tabulación de 32 casos de Embarazo Ectópico Avanzado de acuerdo al lugar de observación, año de observación y año de publicación

(Diversos autores y J. Botero) - Facultad de Medicina - U. de A. 1959

Nombre del autor

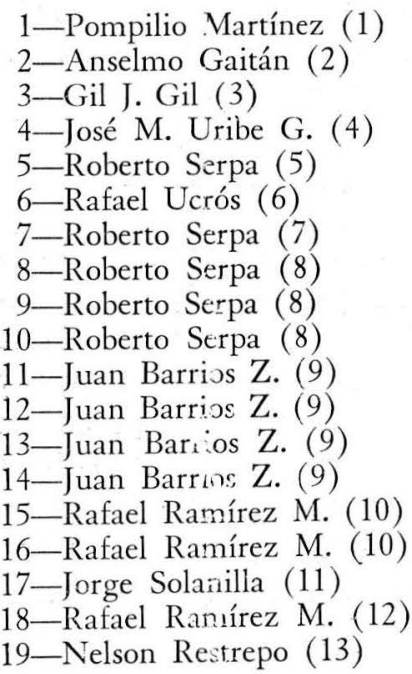

$\begin{array}{lll}\text { Bogotá } & 1911 & 1912 \\ \text { Neiva } & 1912 & 1914 \\ \text { Medellín } & 1916 & 1917 \\ \text { Medellín } & 1921 & 1921 \\ \text { Bucaramanga } & 1923 & 1926 \\ \text { Bogotá } & 1926 & 1927 \\ \text { Bogotá } & 1939 & 1939 \\ \text { Bogotá } & 1915 & 1940 \\ \text { Bucaramanga } & 1919 & 1940 \\ \text { Bucaramanga } & 1917 & 1940 \\ \text { Cartagena } & & 1945 \\ \text { Cartagena } & & 1945 \\ \text { Cartagena } & & 1945 \\ \text { Cartagena } & & 1945 \\ \text { Bogotá } & 1943 & 1946 \\ \text { Bogotá } & 1943 & 1946 \\ \text { Cali } & 1947 & 1950 \\ \text { Bogotá } & 1945 & 1950 \\ & 1948 & \end{array}$




$\begin{array}{llll}\text { 20-Jesús A. Gómez P. (14) } & \text { Bogotá } & 1952 & 1954 \\ \text { 21-Jesús A. Gómez P. (14) } & \text { Bogotá } & 1953 & 1954 \\ \text { 22-Alfredo Londoño U. (15) } & \text { Pereira } & 1953 & 1954 \\ \text { 23-Eduardo Acosta B. (16) } & \text { Barranquilla } & 1956 & 1957 \\ \text { 24-Eduardo Acosta B. (16) } & \text { Barranquilla } & & 1957 \\ \text { 25-Jaime Botcro U. } & \text { Medellín } & 1948 & 1959 \\ \text { 26-Jaime Botro U. } & \text { Medellín } & 1957 & 1959 \\ \text { 27-Jaime Botero U. } & \text { Medellín } & 1958 & 1959 \\ \text { 28-Jaime Botero U. } & \text { Medellín } & 1958 & 1959 \\ \text { 29-Jaime Botro U. } & \text { Medellín } & 1958 & 1959 \\ \text { 30-Jaime Botro U. } & \text { Medellín } & 1959 & 1959 \\ \text { 31-Jaime Botero U. } & \text { Medellín } & 1959 & 1959 \\ \text { 32-Jaime Botero U. } & \text { Medellín } & 1959 & 1959\end{array}$

PRESENTACION DE CASOS

CASO No 1 - E. G., paciente de 27 años de edad, primigesta con fecha de la última menstruación el 5 de Octubre de 1947, admitida al servicio de Obstetricia del Hospital de San Vicente de Paúl el 23 de Diciembre de 1947 con una historia de hemorragia vaginal desde una semana antes, precedida de dolor hipogástrico, vómito, sudoración y pérdida del conocimiento. El abdomen era sensible en el hipogastrio y fosa ilíaca izquierda. Una punción del Douglas permitió obtener sangre oscura. La paciente mejoró en los días que siguieron y fue dada de alta el 11 de Enero de 1948, cuando tenía tres meses de embarazo.

Fue admiticia de nuevo el 4 de Mayo del mismo año, con signos de anemia marcada. Tenía una $\mathrm{Hgb}$. de $4.5 \mathrm{mg} \%$ y un recuento de glóbulos rojos de 1'800.000 por $\mathrm{mm}$. cúbico. Cuatro días después se le trató de inducir el parto, colocándole sondas y balón intrauterino, sin que se consiguiera resultado. Como el estado general empeoraba. 8 días después de admitida se le hizo una laparctomía y se extrajo de la cavidad abdominal un feto vivo de $1.500 \mathrm{gm}$. Con dificultad se hizo extracción de la placenta que estaba parcialmente adherida al fondo del útero, al hígado, colon y epiplón. Se hizo una histerectomía fúndica y se dejaron drenes en la cavidad abdominal La paciente murió al día siguiente debido a reacción producida por una transfusión incompatible, y el niño cinco días más tarde. Este caso se interpretó como un embarazo tubario que en eĩ seguncio mes de su evolución tuvo un aborto tubario con implantación secundaria del embrión en la cavidad peritoneal y ulterior desarrollo allí.

CASO No : - N. O. Paciente de 35 años, grávida 4, para 3, con fecha de la última menstruación en Abril de 1956, fue admitida al servicio de Obstetricia del Hosp̣tal de San Vicente de Paúl el 9 de Abril de 1957, quejándose de dolores abdominales y vómitos. Dos meses después de la última menstruación presentó episodio de dolor abdominal generalizado e intenso que duró dos días aproximadamente y se siguió de mejoría. Desde el cuarto mes de amenorrea observó crecimiento abdominal gradual y desde los ocho meses anorexia, enflaquecimiento y astenia. 
A la admisión la presión arterial era de $100 / 60$, el pulso de 100 por minuto y la temperatura de $37.2^{\circ}$ el aspecto era el de una paciente extremadamente desnuirida con piel seca y descamante y panículo adiposo escaso. Las mamas eran cretantes, el abdomen era globuloso, sensible a la palpación, que demostraka múltiples masas irregulares y duras cuyas dimensiones oscilaban entre 10 y $20 \mathrm{~cm}$. de diámetro, fijas, principalmente localizadas en ambas fosas ilíacas y en la región periumbilical. El examen pélvico demostró que estaban adheridas a las estructuras de la pelvis; el cuello era duro, violáceo, cerrado. Los diagnósticos de impresión fueron: carcinoma de ovario, tumor renal o embarazo abdominal; se tomó una radiografía de abdomen que demostró un feto a término, bien desarrollado, con signos de muerte. Una histerometría practicada dos días más tarde, en el curso de un cuidadoso examen pélvico, fue de $16 \mathrm{~cm}$. y no se encontró obstáculo al paso del instrumento, por lo cual se pensó más seriamente en embarazo abdominal. Una radiografía laterál hecha con el fin de delimitar el contorno uterino, demostró que éste era poco nítido, lo cual también estaba en favor de un embarazo abdominal.

Dos semanas despuás de la admisión, cuando se habían mejorado notablemente la anemia y la desnutrición de la paciente, se hizo un examen bajo anestesia, dilatándose gradualmente el cuello uterino con bujías de Hegar hasta que fue posible introducir el dedo dentro de la cavidad uterina y hallarla vacía. Una biopsia de endometrio tomada en esta oportunidad demostró decidua. Cun el diagnóstico cierto de embarazo abdominal, se procedió a mejorar las co ldiciones nutritivas de la paciente. Cuatro semanas después de la admisión se hizo una laparotomía que demostró un embarazo abdominal con un feto a término macerado bien aislado por una gruesa membrana de tejido fibroso. Se extrajo y se hizo resección parcial de la placenta que se insertaba sobre las estructuras de la pelvis, no permitiendo visualizarlas. Al cerrar la pare ‘ abdominal se déó un dren de penrose comunicado a la cavidad de donce se había extraído el feto. El dren se retiró al 3er. día y la paciente fue dada de alta el $9^{\circ}$, en buenas condiciones.

CASO No 3 - M. R. Z. Paciente de 38 años de edad, grávida 9, para 8, con 11 años de esterilidad secundaria, quien fue admitida por primera vez al servicio de Obstetricia del Hospital de San Vicente de Paúl, el 10 de Diciembre de 1957 por tener un embarazo de tres meses y porque se halló al examen físico una masa bilobulada en el hipogastrio que había producido episodios dolorosos intermitentes. Se hizo entonces el diagnóstico de mioma uterino concomitante con embarazo y se tuvo en reposo por varios días. Mejoraron sus síntomas y se le dio de alta, con instrucciones de regresar a control periódico en la Consulta Prenatal.

Ingresó por segunda vez al servicio el 26 de Marzo de 1958, con una historia de dolores intensos desde 6 días antes localizados principalmente en el epigastrio y en la fosa ilíaca izquierda. El examen físico demostró un abdomen sensible a la palpación, un útero de forma irregular con $35 \mathrm{~cm}$. de altura. La cabeza fetal se palpaba en el epigastrio, al parecer muy superficial; en la fosa ilíaca izquierda se palpaban pequeñas partes y en la fosa ilíaca derecha se palpaba una masa sin forma definida, poco dolorosa, blanda e inmóvil. Una radiografía simple de abdomen demostró un embarazo en el ter- 
cer trimestre, en presentación podálica. Se hizo el diagnóstico presuntivo de quiste de ovario y embarazo abclominal; seis días más tarde se practicó un examen bajo anestesia que demostró un feto que ocupaba el epigastrio y el flanco izquierdo y una masa redondeada y fija, de bordes imprecisos, localizada en el hipogastrio y fosa ilíca derecha; inmediatamente por encima del pubis se palpała el fondo uterine aumentado unas tres veces su tamaño normal, en anteversión y un poco reblandecido. Cuidadosamente se hizo histerometría, que fue de $15 \mathrm{~cm}$. Se confirmó así la presencia de un embarazo abdominal de 29 semanas y se decidió tener la paciente en observación cuidadosa mientras el feto alcanzaba un mayor desarrollo. En los días siguientes la paciente tuvo dolor intermiterite en el abdomen, progresivamente creciente y moderada distensión abdominal. En vista de esto se decidió terminar el embarazo cuando había alcanzade 31 semanas.

23 días deçués de la admisión se hizo una laparotomía y se obtuvo un feto vivo de $2.700 \mathrm{gm}$. de peso que respiró irregularmente a los 15 segundos. Se exploró la cavidad abdominal y se observó que la placenta ocupaba una superficie ampila y se implantabr sobre las estructuras de la pelvis cubriéndolas totalmente y llenando la región supravesical, la fosa ilíaca izquierda y parte de la fosł iliaca derecha. Se seccionó el cordón cerca a su inserción en la placenta y se cerró la cavidad abdominal sin dejar dren. Durante el curso postoperatorio hubo fiebre entre 37 y 38; la placenta se localizó bien y formó una masa hipogástrica remitente que ascendía hasta el ombligo, un poco sensible, pero que nunca dio evidencia de supuración. Nueve semanas después de la intervención fue dada de alta en buenas condiciones. El niño, después de tener un cuadro pulmonar neonatal diagnosticado como bronquitis, y tratado de acuerdo, fue dado de alta con la madre.

Tres meses después de la primera intervención se practicó nueva laparotomía y se encontró la placenta perfectamente diferenciada como una masa quística de paredes muy gruesas, adherida laxamente al fondo de saco de Douglas, a la pared posterior del útero y a asas intestinales. Con facilidad se extirpó el total de la masa placentaria. El útero y los anexos así liberados tenían aspecto normal, excepto la trompa izquierda que era fibrosa y engrosada pero conservaba su longitud total; estos hallazgos sugirieron la posibilidad de un embarazo abdominal primario. Dos semanas más tarde la paciente fue dada de alta en buenas condiciones, después de un postoperatorio sin complicaciones.

En Febrero de 1959, siete meses después de la segunda laparotomía, se intervino de nuevo para corrección de una eventración abdominal. No se exploró la cavidad. Hasta entonces el niño, 14 meses después de haber sido extraído, mostraba un desarrollo satisfactorio.

CASO No 4 - C. T. O. de O (17). - Paciente de 42 años de edad, grávida 5 , para a término 4 , quien consultó a su médico particular por amenorrea de 12 meses, masa abdominal y dolores hipogástricos de pocos días de iniciación. Su embarazo había evolucionado por lo demás normalmente. Una radiografía de abdomen demostró un embarazo aparentemente intrauterino con feto muerto. La laparotomía efectuada en Mayo de 1958 demostró un embarazo abdominal con feto muerto, en buen estado de conservación. Se hizo extracción del feto y de la placenta, sin que se presentara ninguna dificultad. El postoperatorio fue satisfactorio. 
CAso No 5 - C. C. Paciente de 36 años de edad, grávida 13, para 12, con fecha de la última menstruación en Septiembre de 1957, quien fue admitida al servicio de Obstetricia del Hospital de San Vicente de Paúl el 30 de Mayo de 1958, debido a disnea progresiva, edema de miembros inferiores, dolor epigástrico, mareos y cefalea frontal de seis meses de duración. El examen físico en el momento de la admisión demostró una p. a. de 120/80, un pulso de 100 por minuto, pésimo estado general y palidez marcada. El edema de la pared abdominal hacía difícil apreciar el embarazo, que era de 36 semanas. Se hizo el diagnóstico de embarazo e hipoproteinemia. El hemograma mostró una $\mathrm{Hgb}$. de $3.5 \mathrm{gm} . \%$, un Hct. de $12 \%$ y un recuento rojo de $1^{\prime} 600.000$ por $\mathrm{mm}$. cúbico. Las proteínas totales eran de $4.50 \mathrm{gm} . \%$. Durante los días siguientes fue tratado con diuréticos, hierro por vía oral y parenteral, vitaminas y dieta hiperproteica. 26 días después de la admisión la Hgb. había llegado a $5.5 \mathrm{gm} . \%$, el hematocrito a $21 \%$ y el recuento de rojos a $2600.000 \mathrm{x}$ $\mathrm{mm}$. cúbico. Una semana más tarde se iniciaron contracciones de escasa intensidad que no produjeron ningún progreso del parto en 15 horas. El examen pélvico demostró que la cabeza llenaba el fondo de saco de Douglas y no fue posible la individualización del cuello, cuya dilatación no se pudo apreciar. Por falta de progreso en el parto y por no ser posible la apreciación del cuello uterino, se decidió hacer una cesárea. La laparotomía demostró un embarazo abdominal con un feto vivo de $2.900 \mathrm{gm}$. de peso que respiró y lloró vigorosamente dentro del primer minuto que siguió a su extracción. La placenta estaba implantada sobre el fondo de saco de Douglas, el anexo derecho y la cara posterior del útero. Al hacer extirpación parcial de éste se encontró una amplia abertura en su ángulo izquierdo que comunicaba con el saco que contenía el feto, el cual estaba situado entre las hojas del ligamento ancho; esto sugirió que se podría tratar de un embarazo angular o intersticial secundariamente implantado entre las hojas del ligamento ancho. Se hizo una extirpación parcial de la placenta. Para hacer hemostasia se dejaron cuatro tetras llenando totalmente la cavidad pélvica y comprimiendo fuertemente el tejido placentario. La paciente toleró el procedimiento pobremente, perdió unos 2.000 c.c. de sangre y recibió 2.300 . En los tres primeros días del postoperatorio se retiraron gradualmente las tetras sin que se presentara accidente. La herida abdominal drenó líquido serohemático por varios días pero terminó por cicatrizar, dejando una eventración de unos $10 \mathrm{~cm}$. de diámetro. El postoperatorio fue por lo demás satisfactorio; un hemograma hecho 40 días después de la intervención aún demostraba una Hgb. de 4 gm.\% y un Hct. de $19 \%$. El examen físico del recién nacido permitió observar un cráneo alargado en sentido vertical, facies mongoloide, tabique nasal desviado y paladar ojival. Una radiografía de cráneo demostró solamente moderado acabalgamiento occipito-parietal. Mientras estuvo en el hospital progresó satisfactoriamente y fue dado de alta, en buenas condiciones, con la madre.

CASO No 6 - A. Z. de R. Paciente de 42 años de edad, grávida 2, para 1, hace 27 años, quien fue admitida por primera vez al servicio de Ginecología del Hospital de San Vicente de Paúl el 26 de Noviembre de 1958, con la historia de dolor abdominal, endurecimiento del abdomen y metrorragias por un período indeterminado de tiempo. Entre sus antecedentes obstétricos figuraba un embarazo 15 años antes, comprobado radiológicamente, que terminó en muerte fetal y no se siguió de párto; la paciente no quiso someterse al tratamiento sugerido por los médicos y desde entonces había venido observando 
la tumoración causante de los síntomas descritos. Seis meses antes de la admisión había tenido dolor en la región precordial y en el epigastrio, acompañado de sensación de opresión y disnea.

El examen físico demostró ur edema grado II de miembros inferiores, pulso de 100 por minuto, p. a. de 145/105; estertores pulmonares en ambos campos y hepatomegalia. En el hipogastrio y fosa ilíaca derecha había una masa de consistencia dura móvil, cuyas relacones con el útero y los anexos no se pudieron determinar claramente.

Se hizo el diagnóstico de tumor pélvico e insuficiencia cardíaca congestiva y se pidió su traslado al servicio de Medicina Interna, en donde estuvo hospitalizada por espacio de dos meses, al cabo de los cuales se consideró que estaba en condiciones de intervenirse. Mientras tanto una placa simple de abdomen había demostrado un feto muerto y calcáreo en la fosa ilíaca derecha, y en el ovario izquierdo una tumoración calcificada. Una histerosalpingografía demostró la trompa derecha muy dilatada y la izquierda permeable.

Una laparotomía hecha 63 días después de la admisión demostró una masa bilobulada calcificada unida a la trompa derecha; se liberó de sus adherencias y se extirpó con la trompa. Tenía $80 \mathrm{gm}$. de peso y medía $11 \times 18 \times 7 \mathrm{~cm}$.; sobre su superficie había varios huesos largos parcialmente deformados que semejaban un fémur, un húmero y costillas. La masa mayor parєcía corresponder a la cabeza. A la sección se pudo distinguir también la columna vertebral. La paciente fue dada de alta en buenas condiciones en el $7^{\circ}$ día del postoperatorio. Revisada tres meses después, estaba bien.

CASO No y - M. G. L. de R. Paciente de 17 años de edad, grávida 3, para 2, con fecha de la última menstruación desconocida, pero con un embarazo en el último trimestre de su evolución, admitida por tercera vez al servicio de Obstetricia del Hospital de San Vicente de Paúl el 28 de Febrero de 1959, por tener una historia de dos cesáreas anteriores y estar presentando frecuentes dolores abdominales intensos que hicieron sospechar alguna anormalidad del presente embarazo.

Tres años antes de la presente admisión la paciente tuvo eclampsia y su embarazo fue terminado con una cesárea corporal en uno de los municipios vecinos. Un año después, dos antes de la presente admisión, tuvo una ruptura uterina espontánea durante el tercer trimestre de su segundo embarazo y vino a este servicio donde se le practicó una laparotomía y se extrajo un feto muerto. Se hizo sutura de la cicatriz dehiscente después de avivar los bordes.

El examen físico en el momento de la admisión demostró una paciente pálida, en regular estado nutricional, p. a. de 120/80 pulso de 80 por minuto, T. de $38^{\circ}$, altura uterina de $26 \mathrm{~cm}$., ruidos fetales positivos. En los días que siguieron a la admisión, la paciente presentó fiebre persistente, vómito y dolor creciente en el abdomen; los ruidos fetales se hicieron negativos. Diez días después de la admisión se hizo una evaluación cuidadosa de la paciente, que demostró: pulso de 124 por minuto, presión arterial de 115/80, palidez y enflaquecimiento, distensión abdominal y timpanismo, así como signos radiológicos de muerte fetal. Se hizo una punción abdominal y se obtuvo fácilmente 
sangre negra que no coagulaba. Con el diagnóstico de ruptura uterina espontánea por dehıscencia de cicatriz anterior, se practicó una laparotomía. Al abrir el peritoneo se encontró un feto con signos francos de maceración, de $2.000 \mathrm{gm}$. de peso, unido por un cordón infiltrado de sangre a una placenta ampliamente implantada en la porción superior de la pared abdominal anterior, por encima del ombligo, el borde anterior del hígado y algunas asas intestinales delgadas, extendiéndose sobre ellas en un trayecto de unos $10 \mathrm{~cm}$. No había signos de desprendimiento de esta placenta. Se ligó y seccionó el cordón cerca a su inserción en ella y se extrajo el feto. Se exploró luego la cavidad pélvica y se encontró un útero un poco aumentado de tamaño con una dehiscencia en la cara anterior, de una cicatriz previa de cesárea corporal que comprendía todo el eşesor del músculo y se extendía hasta la cavidad, que se encontraba vacía. Las trompas mostraban coalescencia de las fimbrias y aparente obstrucción. Por estos hallazgos y por la edad de la paciente, se decisió dejar el útero, por lo cual se avivaron de nuevo los bordes de la herida y se suturaron con dos hileras de puntos de catgut cromado $N^{\circ} 1$, El curso postoperatorio fue satisfactorio y la paciente dejó el servicio por su propia voluntał en el $12^{\circ}$ día.

CASO No 8 - D. P. C. Paciente de 29 años de edad, grávida 5, para 5 , un gemelar, con fecha de la últina menstruación el 27 de Mayo de 1958 y fecha probable del parto el 6 de Marzo de 1959, quien ingresó por primera vez al servicio de Obstetricia del Hospital de San Vicente de Paúl el 18 de Abril de 1959 con un diagnóstico de feto muerto en presentación transversa. A fines del mes de Febrero, cuando tenía unas 39 semanas de embarazo, tuvo un episodio de dolor epigástrico semejante a trabajo de parto durante unas 5 horas aproximadamente, que se siguió de hemorragia vaginal en cantidad escasa por unas 24 horas; tuvo fiebre por varios días y no volvió a sentir el feto, por lo cual consultó a su médico, quien la envió a este servicio.

El examen físico en el momento de la admisión demostró: p. a. de 130/80, pulso de 120 por minuto, temperatura de $38^{\circ}$; ligero dolor al palpar profundamente los flancos del abdomen. La presentación parecía ser cefálica. En los días siguientes se intentó inducir el parto, administrando estilbestrol, quinina, pitocín diluído en varias ocasiones y finalmente dilatando el cuello uterino e introduciendo sondas vesicales, sin que de ninguna manera se lograra modificarlo. A consecuencia de estas maniobras la paciente desarrolló fiebre de 39.7 ${ }^{\circ}$, que hizo necesario el uso de antibióticos de amplio espectro. 20 días después de la admisión se hizo una laparotomía exploradora: al abrir peritoneo se encontró un feto muerto y macerado que se extrajo, efectuándose a continuación el desprendimiento de la placenta con gran facilidad. El cuerpo uterino era pequeño, de consistencia firme y su cavidad se encontraba en íntima conexión con el saco fetal por una solución de continuidad en toda la anchura de su fondo. Se cerró el peritoneo dejando que el saco drenara por su orificio de comunicación con el útero. El postoperatorio fue satisfactorio; por la vagina drenó un líquido serosanguinolento por varios días que nunca dio evidencias de infección. La paciente fue dada de alta en el $10^{\circ}$ día.

\section{RESUMEN}

Las historias que hemos presentado dan pie para hacer varias consideraciones de interés. 
En cinco casos (Cuadro 2) el embarazo abdominal era el primero que tenía la paciente, y el tiempo transcurrido entre el matrimonio y la iniciación del mismo era menor de 1 año. La mitad de las pacientes había tenido de 1 a tres embarazos; no parece que las grandes multíparas fueran muy predispuestas a sufrirlo.

\section{CUADRO 2}

Graviciez de 32 pacientes con Embarazo Ectópico Avanzado

(Diversos autores y J. Botero) - Facultad de Medicina - U. de A. 1959

Número de Embrrazos

Grávida 1

Grávida 2

Grávida 3

Grávida 4

Grávida 5

Grávida 6

Grávida 7

Grávida 8

Grávida 9

Grávida 13

Multípara, sin especificar

Gravidez desconocida

Totales
Número de casos

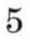

6

5

2

4

1

1

1

1

1

2

3

32
$\%$

15.6

18.7

15.6

6.2

12.5

3.1

3.1

3.1

3.1

3.1

6.2

9.3

100.0

Las pacientes en quienes se obtuvo el dato del tiempo transcurrido desde el último embarazo, constituyeron el $52.9 \%$ del total; en un $34.2 \%$ del mism' total, había transcurrido más de un año desde el último embarazo, y en un $18.7 \%$, incluyendo las primíparas, el tiempo transcurrido era menor de un año. (Cuadro 3 ).

El cuadro 4 muestra los meses de amenorrea, que en la mayoría de las pacientes estaban alrededor del término del embarazo.

No parece que el episodio doloroso en el primero o segundo trimestre sea un signo muy característico, pues dejaron de presentarlo un $40.6 \%$ de las pacientes; naturalmente no sabemos si quien hizo la historia puso especial énfasis en averiguar este punte. 


\section{CUADRO 3}

Tiempo transcurrido desde el último embarazo en 32 casos de Embarazo Ectópico Avanzado

(Diversos autores y J. Botero) - Facultad de Medicina - U. de A. 1959

Tiempo en años

Menos de 1 año

De 1 a 4 años

De 5 a 9 años

De 10 a 14 años

De 15 a 19 años

De 20 a 30 años

Primer embarazo

Desconocido

Totales
Número de casos

$\%$

2

6.2

4

12.5

2

6.2

2

6.2

3.1

6.2

12.5

46.8

32
100.0

\section{CUADRO 4}

Meses de Amenorrea en 32 pacientes con Embarazo Ectópico Avanzado

(Diversos autores y J. Botero) - Facultad de Medicina - U. de A. 1959

Meses de Amenorrea

7

8

9

10

11

12

13

18

20

Desconccido

Totales
Número de casos

1

4

13

2

4

3

2

1

1

1

32

\section{$\%$}

3.1

12.5

40.6

6.2

12.5

9.3

6.2

3.1

3.1

3.1

100.0

(Cuadro 5). Lo mismo puede decirse respecto a la presencia o ausencia de dolores tipo parto y hemorragia vaginal en el tercer trimestre. (Cuadro 6) 


\section{CUADRO 5}

Frecuencia de la aparición de episodio doloroso en el primero o en el segundo trimestre en 32 casos de Embarazo Ectópico Avanzado

(Diversos autores y J. Botero) - Facultad de Medicina - U. de A. 1959 Número de casos $\%$

Episodio doloroso en el prirner trimestre $9 \quad 28.1$

Episodio doloroso en el segundo trimestre

No presentaron episodio dcloroso

Totales

\section{CUADRO 6}

Presencia o ausencia de dolores tipo parto y de hemorragia vaginal a término en 32 casos de Fmbarazo Ectópico Avanzado

(Diversos autores y J. Botero) - Facultad de Medicina - U. de A. 1959

$\begin{array}{lcccc} & \text { Presentes } & \text { Ausentes } & \text { Desconocido } & \text { Total } \\ \text { Dolores tipo parto } & 17 & 10 & 5 & 32 \\ \text { Hemorragia } & 10 & 17 & 5 & 32\end{array}$

A pesar de que en alg inas ocasiones (10, y casos 7 y 8 de esta presentación) se presentaron al mismo autor dos casos consecutivos de embarazo abcominal con muy corto intervalo de tiempo, no se hizo en ninguno el diagnóstico correcto; sin embargo en conjunto sí fue hecho acertadamente en el $37.5 \%$ de los casos, lo cual es un buen índice si se tiene en cuenta la rareza de la entidad. (Cuadro 7). El diagnóstico de embarazo extrauterino con feto vivo se hizo correctamente en tres casos.

\section{CUADRO 7}

Diagnóstico preoperatorio en 32 casos de Embarazo Ectópico Avanzado (Diversos autores y J. Botero) - Facultad de Medicina - U. de A. 1959

$$
\text { Número de casos \% }
$$

Embarazo extrauterino

Embarazo intrauterino

Embarazo intrauterino y otra condición asociada Mioma uterino 
Dos de los casos que hemos publicado ocurrieron en pacientes a quienes se había practicado una cesárea corporal anteriormente, por lo cual debe incluírse ésta entre las causas predisponentes de embarazo abdominal. El caso $\mathrm{N}^{0}$ 7, en el cual había tal situación, lo hemos interpretado como uno de implantación intrauterina originalmente, que fue expulsado por la cicatriz dehiscente a la cavidad abdominal y se implantó en ella en forma secundaria.

Dos de los casos que se encontraron correspondían a embarazos ováricos, pero solamente uno de ellos fue demostrado por estudio anatomopatológico. (Cuadro 8).

De los seis casos en que se obtuvo un feto vivo (Cuadro 9) en dos se dejó la placenta y la paciente sobrevivió; en tres se extrajo y solo una paciente sobrevivió. En el caso restante no se sabe si la placenta fue extraída, pero la madre murió. Parece pues que la extracción de la placenta, si no hay facilidades de transfusión de sangre, tiene influencia decisiva en la determinación de la mortalidad materna.

\section{CUADRO 8}

Clasificación de 32 casos de Embarazo Ectópico Avanzado de acuerdo al sitio de implantación

(Diversos autores y J. Botero) - Facultad de Medicina - U. de A. 1959

Sitio de implantación Número de casos $\quad \%$

Embarazo abdominal, sin más especificación

12

37.5

Embarazo tubario

Embarazo abdominal secunciario

Embarazo intraligamentoso

Embarazo tubo-ovárico

Embarazo orárico (1)

Embarazo t:!bo-intersticial

Localización no especificade.

Totales

(1) Demostrado et. un caso por estudio anatomopatológico. 


\section{CUADRO 9}

Estado del feto en 32 casos de Embarazo Ectópico Avanzado (Diversos autores y J. Botero) - Facultad de Medicina - U. de A. 1959

Estado del feto

Feto vivo

bien ronservado

Feto muerto macerado no especificado

Litopedion

Papiráceo

Totales
Número de casos

5

5

9

7

4

1

32
$\%$

15.6

15.6

28.1

21.8

12.5

3.1

100.0

La mortalidad materna es del $15.6 \%$, lo cual nos parece una cifra muy baja si se tiene en cuenta que la mayoría de los casos ocurrieron antes de que la transfusión sanguínea hubiera alcanzado a usarse con la frecuencia y seguridad con que se hace actualmente. (Cuadro 10).

\section{CUADRO 10}

Mortalidad materna en 32 casos de Embarazo Ectópico Avanzado (Diversos autores y J. Botero) - Facultad de Medicina - U. de A. 1959

$$
\text { Número de casos }
$$
$\%$

Madres muertas 5 15.6

Madres que sobrevivieron

\section{CONCLUSIONES}

Se hace una revisión de la literatura colombiana sobre embarazo ectópico avanzado utilizando el material bibliográfico de la biblioteca de la Facultad de Medicina de la Universidad de Antioquia y se encuentra que hasta el presente se han publicado 24 casos.

Se agregan ocho casos nuevos recopilados en el servicio de Obstetricia del Hospital de San Vicente de Paúl y en el Instituto de Anatomía Patológica de esta ciudad, en los últimos 11 años.

Se hacen algunas consideraciones y comentarios sobre unos de los casos y se intenta una interpretación estadística de algunos datos, a pesar de lo relativamente pequeño de la muestra. 
Se tiene la impresión de que esta recopilación es incompleta, pero se espera que estimule la publicación de otros trabajos que la adicionen.

\section{BIBLIOGRAFIA}

1 MARTINEZ, P.: Un caso de preñez extrauterina (tubaria), de trece meses de duración. Revista Médica de Bogotá. Pág. 129 (Julio) 1912.

2 GAITAN, A.: Un caso raro de embarazo extrauterino. Revista médica de Bogotá. Pág. 520 (Sept-Oct.) 1914.

3 GIL, G. J. Dos casos de preñez ectópica. Revista clínica. Medellín. Pág. 222 (Junio) 1917.

4 URIBE, J. M.: Hospital de San Juan de Dios. Clínica Quirúrgica. Observación sexta. Revista Clínica. Medellín. Pág. 282 (Agosto) 1921.

5 SERPA, R.: Un caso de embarazo extrauterino. Repertorio de medicina y cirugía. Bogotá. 17:547 (Julio) 1926.

6 UCROS, R.: Litopedio salido a través de la cicatriz de una operación cesárea. Revista Médico-Quirúrgica de los hospitales. Bogotá. 2:405. (Febrero) 1927.

7 SERPA, R.: Un caso de embarazo extrauterino a término. Boletín de la Clínica de Marly. Bog tá. 1:98 (Dic.) 1939.

8 SERPA, R.: El embarazo extrauterino. Recuento general. Rev. Fac. de Med., Bogotá. 8:455 (Mayo) 1940.

9 BARRIOS, J.: Embarazo tubo-abdominal a término. Rev. de Med. y Cir. Barranquilla. 12:48 (Sept.) 1945.

10 RAMIREZ, R. F.: Embarazo ectópico casi a término. Relato de dos historias clínicas. Med. y Cir., Bogotá. 10:307 (Junio) 1946.

11 SOLANILLA, J.: Citado por Navas (18).

12 RAMIREZ, R. F.: Citado por Navas (18).

13 RESTREPO. N.: Citado por Navas (18).

14 GOMEZ, J. A.: Noventa y cinco casos de embarazo ectópico. Rev. Fac. de Med., Bogotá. 22:351 (Abril) 1954.

15 LONDOÑO, A.: Una historia de embarazo abdominal. Revista Colombiana de Obstetricia y Ginecología. 5:205 (Marzo-Abril) 1954.

16 ACOSTA, E.: Comentarios acerca de dos casos de embarazo ectópico a término. Revista Colombiana de Obstetricia y Ginecología. 8:195 (Julio-Agosto) 1957.

17 HENRIQUEZ, V.: Comunicación personal al autor.

18 NAVAS, G.: Casos curiosos de embarazo ectópico avanzado. Revista Colombiana de Obstetricia y Ginecología. 1:144 (Junio-Julio) 1950. 